\title{
On the Comparison of Two Vehicular Safety Systems in Realistic Highway Scenarios
}

\author{
A. Amoroso, G. Marfia, M. Roccetti, C. E. Palazzi* \\ Computer Science Department, University of Bologna, Bologna, Italy \\ *Pure and Applied Math Department, University of Padua, Padua, Italy \\ E-mail: \{amoroso, marfia, roccetti\}@cs.unibo.it, cpalazzi@math.unipd.it \\ Received May 13, 2011; revised June 15, 2011; accepted July 3, 2011
}

\begin{abstract}
The application of wireless VANET technology to accident warning systems is gaining an increasing interest. These systems can significantly increase the safety of daily driving and are based on a technology that is steadily becoming mature. We present an experimental comparison between two effective approaches that cope with realistic scenarios. Both rapidly broadcast alert messages throughout platoons of vehicles, and are based on wireless vehicle-to-vehicle (V2V) communications. However, with one approach an alert message propagates through the farthest relay at each hop, whereas with the other it propagatesusing the farthest spanning relay (i.e., the relay that can retransmit farthest away an alert message). With this study we will see retransmitting through the farthest spanning relay at each hop can improve the performance by a factor of two in terms of propagation delay, in comparison to choosing the farthest relay.
\end{abstract}

Keywords: Intelligent Transportation Systems, Accident Warning System, VANET

\section{Introduction}

A promising usage of emerging new technologies is the application of inter-vehicular communications to significantly improve vehicular safety in highway scenarios. In fact, an effective warning system between vehicles could be built either by means of pre-installed infrastructures, or by means of wireless vehicle-to-vehicle (V2V) technologies. The latterapproach, however,appearsmore feasible, less costly and more efficient on a whole network of highways. Warning systems based on V2V technologies offer good performance and effectiveness, as alert messages are directly broadcast between vehicles and do not experience the delay of flowing through a centralized server. Therefore, both the academic research and the industries are proposing several safety systems based on V2V technologies.

As a result, it is now a widely shared idea that effective vehicular warning systems could be built upon integration between vehicular ad-hoc networks (VANETs) and pervasive sensor technologies, as the most promising VANETs are based on the standard IEEE 802.11p [1-5]. The 802.11p standard defines V2V communications that do not rely on any infrastructure, and that are built by means of wireless devices installed on vehicles.
However, a few common proposals are also based on 3G technologies (e.g., smartphones). To summarize, these proposals usually assume that a smartphone equipped with an accelerometer (or similar technologies) is on board ofvehicles. In case an accident occurs, generating an abnormal acceleration, the smartphone (i.e., the accident warning software running on it) sends an alert message to a centralized server, via the cellular infrastructure. In turn, the centralized server advertises such event to all overcoming vehicles, via FM radio or again via the cellular infrastructure, for example.

Although cellular-based solutions are theoretically feasible, the results that have been so far presented suggest that they cannot match the requirements posed by efficient accident warning system [6,7]. An accident warning message of a few kilobytes, transmitted between two moving vehicles through a cellular connection could often experience latencies in the order of several seconds, thus resulting almost useless in this scenario.

Hence, several proposals have been so far presented in the scientific literature to rapidly broadcast alert messages among vehicles, by means of V2V communications; these proposals are too many to be cited.However, most of them do not take into account the following characteristics of realistic scenarios: 
- Different vehicles could have different transmission ranges;

- The transmissions ranges of each vehicle could change while traveling.

As a consequence of these characteristics, it might happen that vehicles experience asymmetric communications: a given vehicle could receive messages from another vehicle but not vice versa. To face suchproblem, a new class of V2V algorithms to spread alert messages is emerging, aimed at optimally transmitting alert messages, while taking into account the above-mentioned realistic conditions.

The purpose of this paper is the comparison of two among the most effective V2V algorithms that work under realistic assumptions [2,8].The first one adopts the strategy of propagating alert messages through the farthest relay, while the second chooses the farthest spanning relay. To clearly understand the performance differences, in terms of propagation delay, that these two choices entail, we compared both protocols under the same experimental conditions in wide set of simulations and different propagation scenarios. Anticipating here our results, using the farthest spanning relay algorithm it is possible halve the time require to disseminate alert messages, compared to using the farthest relay.

It goes without saying that our comparison could include any new proposals, as these will meet the same aims.

The remainder of this paper is organized as follows. The next Section outlines the motivations of our work. Section 3 describes which are the main challenges in VANET highway scenarios, while Section 4 provides a short description of the main ideas behind the two algorithms that we compare. Section 5 describes the main experimental results that we acquired by means of our extensive simulation analysis. To end, Section VI contains some concluding remarks.

\section{Motivation}

We discuss in the following example the positive effect that VANET-based technologies may have in ensuring vehicular safety. The adoption of VANET-based accident warning system reduces the number of vehicles that could potentially be involved in an accident.

Specifically, we simulated a vehicle crash on a threelane highway and estimated how many vehicles could, on average, be involved in it. In the simulation, we accounted for both realistic driver response times, and vehicles lengths. According to the measurements reported in [9] and [10], drivers' response times were randomly drawn from the $(0.75,1.4)$ s range, while vehicle lengths from the $(3.5,5) \mathrm{m}$ interval. Hence, in our simulation model, just as it happens in reality, a driver waits before braking, for a time equal to his/her response time, after realizing that the vehicle that immediately precedes him/her is braking.

We compared the amount vehicles involved in two scenarios: with and without an accident warning system in place. Both scenarios were simulated in the cases of congested and not-congested traffic flows. Additionally, we also considered wet and dry pavement conditions (i.e., different friction constants).

Finally, to be as realistic as possible, vehicle speeds and related time-headway distributions were drawn from real measurements [11]. Specifically, in the uncongested scenario, vehicles moved at $110 \mathrm{~km} / \mathrm{h}$ and their timeheadway distribution caused a linear density of 20 vehicles per $\mathrm{km}$, on a per lane basis. In the congested scenario, speed was on average equal to $40 \mathrm{~km} / \mathrm{h}$ and the linear density of vehicles was of 40 per kilometer. The kinetic friction constant between the tires and the asphalt was equal to 0.2 when wet, and to 0.8 when dry, according to [12].

We ran 100 simulations for each one of the 8 different scenarios described above. The average numbers of crashed vehicles involved in an accident are shown in Figure 1, with their related 95\% confidence intervals. The darkest bars represent the average number of vehicles involved in an accident in case only the traditional warning system was used (i.e. drivers alerted by the preceding vehicles brake lights), while the light gray bars represent the cases where a VANET-based accident warning system was running.

Firstly, let us focus on the contexts when the pavement is dry, thus when braking is more effective (first and third pair of bars in Figure 1). When the pavement is dry, the deployment of VANET-based accident warning systems reduces the average number of crashed vehicles by nearly the $40 \%$. In the worst case, instead, which occurs when the pavement is wet, the average benefit deriving

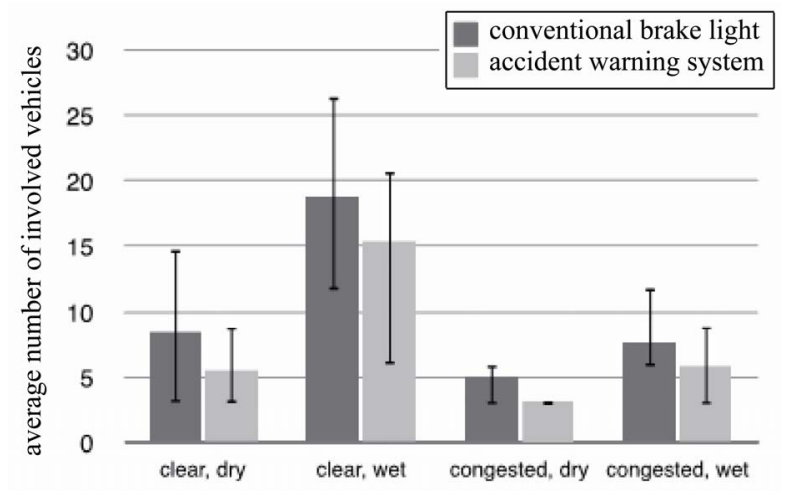

Figure 1. Average number of vehicles involved in an accident. 
from the adoption of a VANET-based accident warning system amounts to about 25\%; this still represents a significant result.

From the above simulation results, it should be clear that VANET-based alert systems could provide valid solutions to accident warning challenges. The adoption of such solutions could soon become a reality as it is reasonable to assume that in the next few years a large number of vehicles will be equipped with both GPSbased navigation systems and 802.11 p networking capabilities. In [13] the author reports the most prominent ongoing projects in this field carried by major car manufacturers.

\section{Challenges in Highway Scenarios}

As widely discussed in literature, there are several differences between VANETs and traditional ad hoc networks $[3,14,15]$; the main differences could be summarized as follows:

- Higher speeds in vehicular environments than in traditional ad hoc networks;

- Vehicles typically move along one-dimensional topologies in highway scenarios, whereas nodes usuallymove along bi-dimensional trajectories in typical ad hoc network scenarios (e.g., military).

Moreover, due to their high speeds, vehicles are subjected to highly varying surroundings that affect their transmission ranges. As an example, consider a vehicle following a long truck; in such case the forward transmission range of this vehicle is severely reduced while remaining behind the truck. When the same vehicle overtakes the truck, its forward transmission range abruptly increases, while its backward transmission range could instead experience the inverse phenomenon. In a similar manner, several events can affect the transmission ranges of vehicles while driving along a highway. As examples consider: tunnels, road dips and hills, surrounding buildings and trees, weather conditions, and so on.

For these reason, in this work we compare two accident warning system proposals that take into account the fact that the transmission ranges of vehicles can radically change in time at a fast pace, while travelling in a highway scenario.

In fact, under the realistic assumptions of different and varying transmission ranges, many accident warning system proposals might not exhibit satisfactory performances, or even not work at all $[4,16]$. The rationale for the poor performances of many proposals depends on many different factors:

- Transmission ranges are not constant in time;

- Communications can be asymmetric;

- Global network topology knowledge is often unfea- sible to obtain.

Recently, new approaches emerged to rapidly broadcast alert messages; these new methods account for the realistic assumption that transmission ranges may be anything, but equal and constant in time.

Among these methods, we have considered as promising the one presented in [2]. This proposal attains very fast broadcast of alert messages by minimizing the number of hops, i.e. minimizing the number of vehicles that relay the alert message. Its intuition is that the farthest vehicle that receives an alert message should relay it; a lightweight mechanism selects the farthest receiver of each message. To do so, this approach estimates the actual transmission range of each vehicle.

Under the same realistic assumptions, an additional method that effectively minimizes the number of hops has been proposed in [8]. To achieve such result, the latter scheme chooses as a relay, among all the vehicles that receive an alert message, the vehicle whose re-transmission will span farthest away in space (i.e., the farthest spanning relay).

\section{Choosing the Best Relay in Realistic Scenarios}

In this Section we briefly outline the main ideas behind the two chosen protocols, namely [2] and [8]. It is worth noticing that we are not interested in providing in this paper an exhaustive discussion of all the technical details underlying these protocols, as these can be found in the referenced papers. Rather, we want to succinctly discuss their most prominent characteristics.

Before proceeding, we anticipate here that both protocols assume that GPS and navigation data are locally available on each vehicle. Moreover, to build the local knowledge they utilize, both protocols share the same technique of exchanging utility messages between neighboring vehicles within a given platoon. Finally, accidentwarning messages are in general asynchronous with respect to utility messages and both of the chosen protocols append some data to the these messages in order to choose the best relay.

\subsection{Farthest Relay}

Let us start with [2] that selects as its best relay, at each step, the farthest receiver of a message. This method relies on two relevant features: the assessment of the transmission ranges, and the probabilistic mechanism of the re-transmission procedure.

Each utility message sent by a vehicle contains itsupdated position and the IDs of the set of all vehicles 
whose transmissions have been recently received by that given vehicle. Upon receiving a utility message a vehicle can estimate its transmission range computing its distance from the sender of that utility message. Assuming that utility messages are frequent enough, each vehicle can be aware of all the vehicles that have received its recent transmissions. Obviously, this mechanism assumes that communications are symmetric.

When the accident-warning software running on a vehicle generates an alert message, it also appends to that alert message the estimated transmission ranges of the vehicle. This information, in turn, is used by all the vehicles that receive that message to determine if they are near to (or far from) the boundaries of the transmission range of the vehicle that generated that alert. Utilizing such information, each vehicle computes a contention window for the re-transmission of the alert message, that is inversely proportional to its distance from the sender of that alert. The closer a vehicle is to the boundary of the transmission range, the shorter its contention window and the higher the probability that it will act as the relay. This mechanism has also the advantage of reducing the possibility of collision between relays. In fact, each vehicle waits a random time inside its contention window before re-transmitting the message, and the re-transmission of a message takes place solely if none of the other vehicles did it before.

Unfortunately, if transmission ranges are different on a per vehicle basis, choosing as a relay the farthest vehicle among all that receive a given message is not the best strategy to minimize the number of hops. It could happen, in fact, that the relay has a shorter transmission range in comparison to that of another vehicle, traveling in between the sender and the relay. Hence this second vehicle could span the alert farther than the relay that the discussed method has chosen. In such case, the proposed algorithm would select a sub-optimal relay.

\subsection{Farthest Spanning Relay}

To optimize the number of hops, the method proposed in [8] selects as the best relay of an alert message that vehicle, among all the receivers, whose re-transmission will span farthest. This approach really achieves the results of minimizing the number of involved hops.

This result is achieved through different modifications to the scheme of the previous algorithm, the first of which is the idea of better exploiting the information on transmission ranges, which is now inserted in the utility messages. Hence, each vehicle of a platoon sends utility messages to inform its peers of: its position, its transmission range, and the IDs of the set of vehicle from which it has recently heard communication messages. Upon receiving these utility messages, each vehicle can compute an updated estimation of its own transmission range and also obtain the ID of the set of receivers of its messages plus an estimation of their correspondent transmission ranges. All this can be used to overcome the problem of asymmetric communications.

Suppose, in fact, that vehicle $r$ hears vehicle $s$, but not vice versa. As all vehicles now receive utility messages containing the set of information mentioned above, a given vehicle $i$ could exist, between $r$ and $s$, that is able to detect this anomaly in the communication between the two peers, as it hears the utility messages from both $r$ and $s$. At this point the problem is sorted out as vehicle ican add information about $r$ in its utility messages so that when $s$ receives them it candiscover that its messages span till $r$.

By virtue of the mechanism mentioned before, the activity of broadcasting accident alert messages works as follows. Any vehicle in the situation of generating an alert message appends to it a list of possible relays, ordered based on the length of their re-transmission span. This is possible only because utility messages transport information concerning the transmission ranges of the vehicle that have emitted those utility messages. As soon as a certain vehicle receives an alert message, it waits a time proportional to its position in the list of relays before re-transmitting the message. If none did it before, the vehicle retransmits the alert message. Note that the horizon of knowledge of each vehicle contains solely its neighbors.

Clearly, the advantages that this scheme provides come at an increased overhead cost, compared to its competitor. In particular, authors of [2] show that their scheme can operate with less than $1 \mathrm{~kb} / \mathrm{s}$ of utility messages within a transmission area, opposed to $75 \mathrm{~kb} / \mathrm{s}$ required by [8] in normal situations.

\section{Simulation Assessment}

For the sake of conciseness, we will refer to the aforementioned algorithms by using the original names, which were given by their designers. Hence, we denote with PIVCA the algorithm that selects the farthest relay [2], while we denote with FROV the one that exploits the farthest spanning relay [8].We evaluated the performance of PIVCA and FROV against three different parameters: 1) end-to-end delay, 2) number of hops, and 3) number of lost messages.

Propagation times and the number of involved hops, in particular, represent the most important figures of merit as to the efficacy of the examined approaches. The number of lost messages accounts instead for the reliability of the methods, since none of them exploits ACK based 
mechanism to guarantee that all the cars in a platoon have received the warning of an accident.

We used Ns2 [17] to run our simulations, considering alert messages of 1 Kbyte sent within two different scenarios:

- Transmission ranges of vehicles remain constant during the simulation;

- Transmission ranges of the vehicles change during the simulation, as it happens in realistic situations.

\subsection{Constant Transmission Ranges}

The simulation considered a platoon of 400 vehicles on an $8 \mathrm{~km}$ long portion of a single lane road. At the beginning of the simulation, we considered the road as divided in slots, each of $20 \mathrm{~m}$ in length. We randomly placed one vehicle in each slot.

Each of the 400 vehicles moved at a constant, but different speed. In literature, it is shown that the distribution of the speeds of vehicles on a freeway is a bell shaped around a median value [18]. Therefore, we randomly set the constant speeds of the 400 vehicles of our experiments as shown in Figure 2.

Each vehicle had a forward (and a backward) transmission range randomly chosen in the $(100,600) \mathrm{m}$ interval.

To measure propagation times, we considered two probe vehicles: the first vehicle was at the beginning of the platoon while the second vehicle was at the end of the platoon. These specific vehicles constantly remained at a distance of $8 \mathrm{~km}$ one from other, for the entire simulation period. All measurements were taken with respect to these two vehicles.

To put our system under severe stress, we supposed that more than one vehicle could send an alert message. We tested the cases of 1, 20, 40, 60, 80, 100 different senders, respectively, randomly chosen within the platoon. Obviously, any vehicle could send an alert message

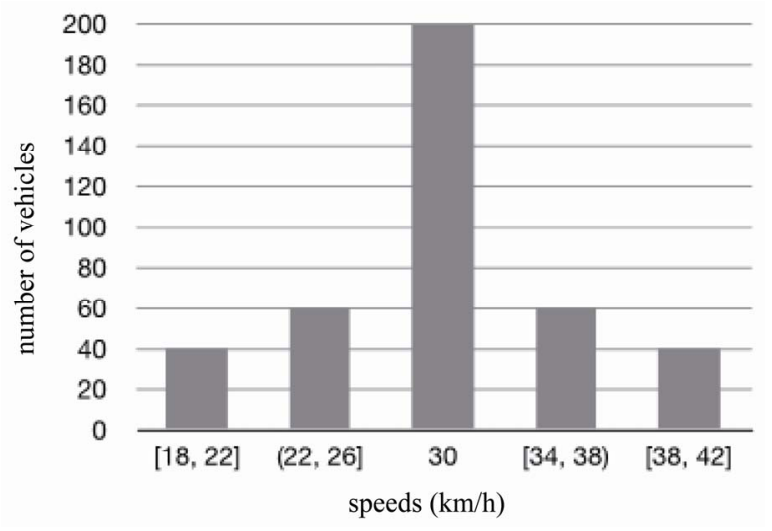

Figure 2. Speed distribution.
We tested the cases of 1, 20, 40, 60, 80, 100 different senders, respectively, randomly chosen within the platoon. Obviously, any vehicle could send an alert message, independently of other vehicles. Moreover, the sending vehicles repeatedly sent alert messages on a periodical basis, i.e. they sent alert messages randomly within a period of $(1,1.5) \mathrm{s}$.

\subsubsection{Propagation Time}

We repeated each simulation ten times, varying the random seed at every new run. Figure 3 shows the average propagation times, in milliseconds, for each different case. From a first inspection of that figure, it clearly emerges that FROV is at least twice as faster than PIVCA.

We consider that each broadcast terminates when the alert message reaches all the vehicle of the platoon, i.e. when both of the benchmark vehicles received the alert message.

In particular, the leftmost pair of points in Figure $\mathbf{3}$ shows the case of a single vehicle that sends alert messages. In this case, a FROV message took an average of about $120 \mathrm{~ms}$ to reach all the vehicles of the platoon, while a PIVCA message took more than twice that time (295 ms).The remaining points show the average times taken alert messages when several vehicles act as sources.

It is interesting to note that in the cases of multiple senders both PIVCA and FROV almost do not undergo any performance degradation with respect to the case of a single sender.

FROV assesses quite precisely the transmission ranges of all the vehicles, thus resulting in a higher speed in

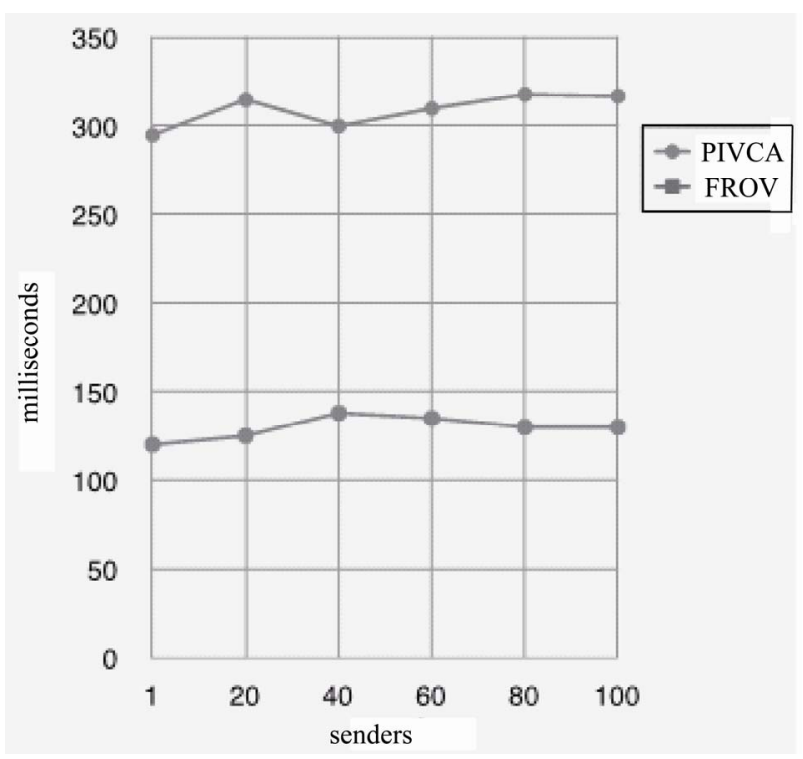

Figure 3. Propagation times. 
reaching all the vehicles in the platoon (by a factor of two at least). This also depends on the optimality of the relay policy that FROV implements.

\subsubsection{Number of Hops}

Figure 4 shows the number of hops required by the broadcasts discussed in the previous section. These results confirm what already observed with propagation times.

Note that, on average FROV takes about 30\% less hops with respect to PIVCA.

The number of hops taken by each broadcast in FROV is almost independent of the number of senders. While the alert messages are independent of each other, their broadcasts take the same number of hops.

\subsubsection{Percentage of Lost Messages}

As already mentioned, neither PIVCA nor FROV guarantee the delivery of each alert message to each vehicle of the platoon, as they do not exploit ACKs. In practice, it might happen that some alert messages get lost, with the possibility that some of the vehicles in the platoon are not informed about an accident. To measure this, Figure 5 reports the average number of lost messages (with their 95\% intervals of confidence) experienced by PIVCA and FROV in the above-described scenarios.

Interestingly, even in the most stressful cases, the number of lost messages was very low. This confirmsit that is not necessary to implement an ACK mechanism to effectively broadcast accident-warning messages.

The simulation shows that the number of lost messages with FROV is directly proportional to the number of senders, and therefore to the number of alert messages to broadcast. The number of messages lost by PIVCA,

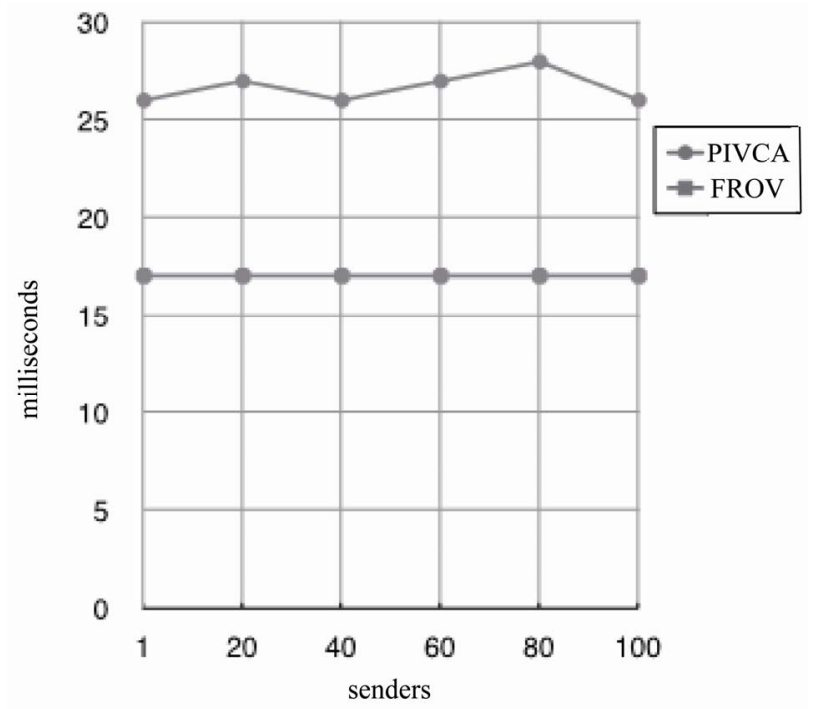

Figure 4. Number of hops.

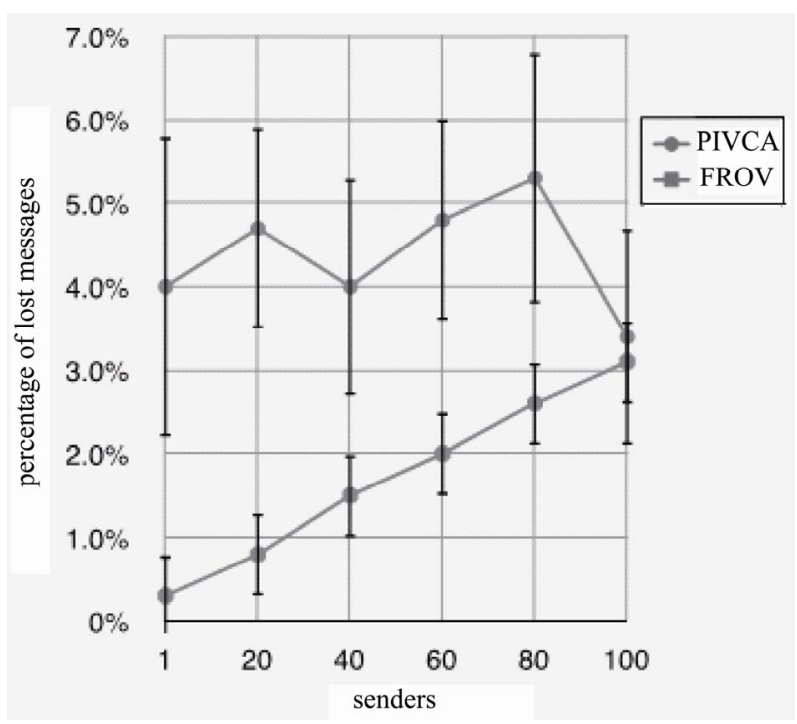

Figure 5. Percentage of lost messages.

instead, seems to be quite independent on the number of broadcasts.

\subsection{Variable Transmission Ranges}

We finally tested the capability of both PIVCA and FROV to adapt to the realistic variations of vehicles' transmission ranges. To do this, we considered a platoon of vehicles traversing a tunnel, factor that may significantly and suddenly reduce the transmission ranges of all the involved vehicles. Hence, to stress this point, we set that as vehicles entered the tunnel, their transmission ranges abruptly halved.

In particular, flowing through the tunnel had the following effects on the transmission ranges of vehicles, depending on their position:

- Entering the tunnel: the portion of the forward transmission range that falls inside the tunnel gets halved. The same event occurs to the backward range,as the vehicle passes the entrance of the tunnel;

- Inside the tunnel: both forward and backward ranges are halved with respect to their initial values;

- Exiting the tunnel: when the vehicle exits the tunnel its forward range suddenly doubles, restoring its initial value. The portion of backward range that still falls inside the tunnel remains halved with respect to its initial value.

We simulated a platoon of 100 vehicles. At the beginning of the experiments, we distributed the vehicles of the platoon on a portion of road that was $2 \mathrm{~km}$ long, following the same scheme discussed in Section V.A. The speeds of the vehicles were chosen based on the same distribution mentioned above. In this set of experiments, the benchmark vehicles were initially at the two ends of 
the platoon, i.e. they remained about $2 \mathrm{~km}$ apart during the experiment. The tunnel was $1 \mathrm{~km}$ long and at the beginning of the experiments it was ahead of the first benchmark vehicle.

We limited to 10 the number of vehicles that sent alert messages; each one of these vehicles implemented the sending scheme discussed above.

\subsubsection{Propagation Time}

We repeated a set of 10 experiments for each case, varying the random seed each time. Figure 6 shows the average propagation times for both PIVCA and FROV. We reported the usual 95\% intervals of confidence atop of each bar. The Figure shows two different cases: when the platoon traverses a clean portion of road, i.e. without the tunnel, and when the platoon traverses the tunnel. In the latter case, the experiment was long enough to allow both the benchmark vehicles to traverse the tunnel. Measurements were taken since the moment when the first benchmark vehicle entered the tunnel, till the moment when the second benchmark vehicle left the tunnel. We computed the average time taken by alert messages to reach both the benchmark vehicles. Figure 6 shows two important results: 1) FROV is remarkably faster than PIVCA in any circumstance, but 2) it suffers the tunnel much more than PIVCA. In other words, FROV experiences a loss of performance close to $30 \%$, when the platoon passes through the tunnel. Our insight here is that the relative loss of performance of FROV is mainly caused by the mechanism of assessment that takes some time to detect the reduction of the transmission ranges. In fact, a vehicle would probably insert in the list of possible relays several other vehicles that, instead, are no longer reachable. This leads some vehicles to erroneously consider their transmission ranges longer than in reality. Thus, as soon as the mechanism of transmission

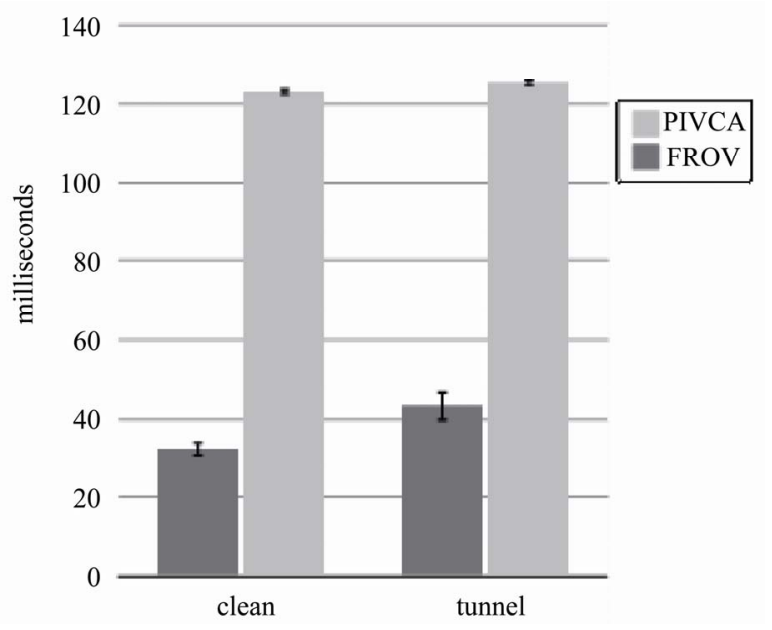

Figure 6. Propagation times with and without the tunnel. range assessment updates its values, FROV returns to operate as usual. PIVCA, instead, suffers less the perturbations of trans- mission ranges brought by the tunnel. This effect depends on the intrinsic nature of PIVCA, which ignores asymmetric communications, thustheir disruption of them does not cause any interference.

\subsubsection{Number of Hops}

In contrast with the measurements taken for propagation times, the average number of hops appears to remain quite stable even in the presence of the tunnel for both PIVCA and FROV. Figure 7 shows the average number of hops we measured in all the examined cases.

\subsubsection{Percentage of Lost Messages}

Figure 8 shows the percentage of messages lost with PIVCA and with FROV, respectively, in the presence of the tunnel. While with a clean road the performance of FROV are one order of magnitude better than PIVCA, the presence of the tunnel causes FROV to lose about ten times more messages. This causes the fact that, when the platoon traverses the tunnel, the average number of lost messages by PIVCA and by FROV is basically the same. In any case, the percentage of lost messages is still very low, thus confirming the fact that these methods are reliable enough even without exploiting ACK-based mechanisms. Again the sharp increase of lost messages experienced by FROV depends on the temporary flawed gauges of the assessment mechanism, which suffers when vehicles enter and exit the tunnel.

\section{Conclusions}

\subsubsection{Percentage of Lost Messages}

Figure 8 shows the percentage of messages lost with PIVCA and with FROV, respectively, in the presence of

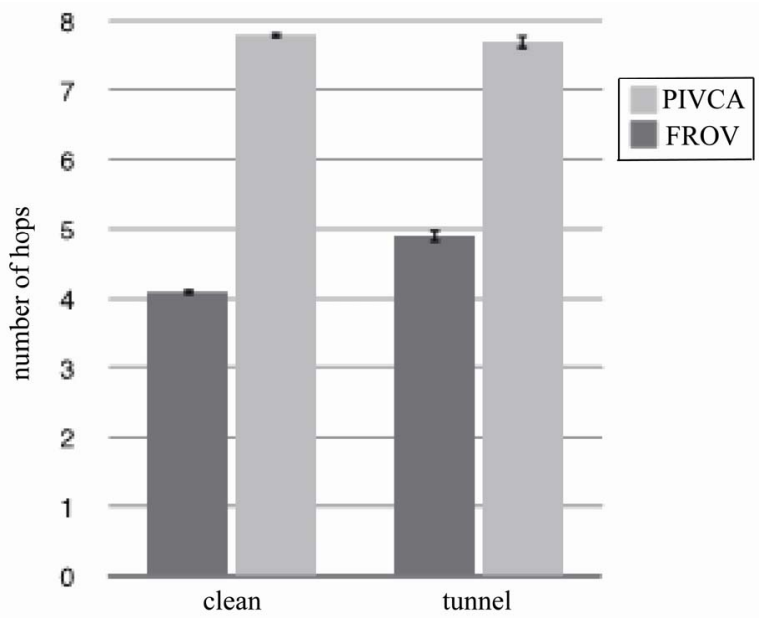

Figure 7. Number of hops with and without the tunnel. 
the tunnel. While with a clean road the performance of FROV are one order of magnitude better than PIVCA, the presence of the tunnel causes FROV to lose about ten times more messages. This causes the fact that, when the platoon traverses the tunnel, the average number of lost messages by PIVCA and by FROV is basically the same. In any case, the percentage of lost messages is still very low, thus confirming the fact that these methods are reliable enough even without exploiting ACK-based mechanisms. Again the sharp increase of lost messages experienced by FROV depends on the temporary flawed gauges of the assessment mechanism, which suffers whenvehicles enter and exit the tunnel.

\section{Conclusions}

We here described the results of an extensive experimental comparison between two approaches that cope with realistic $\mathrm{V} 2 \mathrm{~V}$ scenarios. Both of these approaches aim at delivering fast broadcast of alert messages by minimizing the number of retransmission. However, the simulation results have shown that FROV achieves the best performances in terms of dissemination delay, at a cost of a higher, but feasible, amount of protocol overhead.

\section{Acknowledgements}

The Italian FIRB DAMASCO funded this work.

\section{References}

[1] G. Korkmaz, E. Ekici, F. Ozguner and U. Ozguner, "Urban Multi-Hop Broadcast Protocol for Inter-Vehicle Communication Systems," Proceedings of the 1st ACM International Workshop on Vehicular Ad Hoc Networks, ACM, New York, October 2004, pp. 76-85.

[2] C. E. Palazzi, M. Roccetti and S. Ferretti, “An Intervehicular Communication Architecture for Safety and Entertainment," IEEE Transactions on Intelligent Transportation Systems, Vol. 11, No. 1, 2010, pp. 90-99. doi:10.1109/TITS.2009.2029078

[3] N. Wisitpongphan, O. K. Tonguz, J. S. Parikh, P. Mudalige, F. Bai and V. Sadekar, "Broadcast Storm Mitigation Techniques in Vehicular Ad Hoc Networks,” IEEE Wireless Communications, Vol. 14, No. 6, 2007, pp. 84-94. doi:10.1109/MWC.2007.4407231

[4] A. Zanella, G. Pierobon and S. Merlin, "On the Limiting Performance of Broadcast Algorithms over Unidimensional Ad-Hoc Radio Networks," Proceedings of WMPC 04, September 2004.

[5] 802.11 WG, IEEE Std 802.11, 1999 Ed, Part II: Wireless
LAN MAC and PHY Layer Specs, IEEE.

[6] M. Elbes, A. Al-Fuqaha and M. Guizani, “A New Hierarchical and Adaptive Protocol for Minimum-Delay V2V Communication,” Proceedings of the 2009 IEEE Global Telecommunications Conference, November 2009, pp. 1-6. doi:10.1109/GLOCOM.2009.5426112

[7] C. Wewetzer, M. Caliskan, K. Meier and A. Luebke, "Experimental Evaluation of Umts and Wireless lan for Inter-Vehicle Communication Telecommunications," Proceedings of the 7th International Conference on ITS, August 2007, pp. 1-6.

[8] M. Roccetti, G. Marfia and A. Amoroso, "An Optimal 1d Vehicular Accident Warning Algorithm for Realistic Scenarios," Proceedings of IEEE Symposium on Computers and Communications, June 2010.

[9] R. Akcelik, "On the Validity of Some Traffic Engineering Folklore," In Proceedings of 22nd Conference of Australian Institutes of Transport Research, December 2000.

[10] M. Bilban, A. Vojvoda and J. Jerman, “Age Affects Drivers' Response Times,” Journal Collegium Antropologicum, Vol. 33, No. 2, June 2009, pp. 467-471.

[11] L. Neubert, L. Santen, A. Schadschneider and M. Schreckenberg, "Single-Vehicle Data of Highway Traffic: A Statistical Analysis,” Physical Review E, Vol. 60, No. 6, December 1999, pp. 6480-6490. doi:10.1103/PhysRevE.60.6480

[12] C.-G. Wallman and H. Astrom, "Friction Measurement methods and the Correlation between Road Friction and Traffic Safety," Swedish National Road and Transport Research Institute, Technical Representative, 2001.

[13] C. Squatriglia, "Talking Cars Are Coming Soon to Keep Us Safe,” Wired-Autopia, January 2011. http://http://www.wired.com/autopia/2011/01/talking-cars -are-coming-soon-to-keep-us-safe

[14] S. Biswas, R. Tatchikou and F. Dion, "Vehicle-to-Vehicle Wireless Communication Protocols for Enhancing Highway Traffic Safety,” IEEE Communication Magazine, Vol. 44, No. 1, 2006, pp. 74-82.

[15] F. Ye, M. Adams and S. Roy, "V2v Wireless Communication Protocol for Rear-End Collision Avoidance on Highways," Proceedings of IEEE 2008 VehiMobi Workshop, May 2008, pp. 375-379.

[16] J. J. Blum and A. Eskandarian, “A Reliable Link-Layer Protocol for Robust and Scalable Intervehicle Communications," IEEE Transactions on Intelligent Transportation Systems, Vol. 8, No. 1, 2007, pp. 4-13. doi:10.1109/TITS.2006.889441

[17] The Network Simulator-NS2. http:// www.isi.edu/nsnam/ns/.

[18] A. Benjamin, "Empirical Highway Speed Comparison between Germany and the USA," Oregon State University, 2006. 